\title{
Mips: The Multiband Imaging Photometer For SIRTF
}

Rieke, G., Lada, C., Lebofsky, M., Low, F., Strittmatter, P., et al.

G. H. Rieke, C. Lada, M. Lebofsky, F. Low, P. Strittmatter, E. Young, J. Arens, E. Haller, P. Richards, C. Beichman, T. N. Gautier, J. Mould, G. Neugebauer, $S$. Gaalema, M. Werner, "Mips: The Multiband Imaging Photometer For SIRTF," Proc. SPIE 0619, Cryogenic Optical Systems and Instruments II, (18 July 1986); doi: 10.1117/12.966641 


\section{MIPS: The Multiband Imaging Photometer for SIRTF}

G. H. Rieke, C. Lada, M. Lebofsky, F. Low, P. Strittmatter, and E. Young Steward Observatory, University of Arizona, Tucson, AZ 85721

J. Arens, E. Haller, and P. Richards

University of California, Berkeley, CA 94726

C. Beichman and T. N. Gautier

Jet Propulsion Laboratory, $480 \emptyset$ Oak Grove Drive, Pasadena, CA 91109

J. Mould and G. Neugebauer

California Institute of Technology, Pasadena, CA 91125

S. Gaalema

Hughes Aircraft Company, Carlsbad, CA $920 \emptyset 8$

M. Werner

NASA Ames Research Center, Moffett Field, CA 94035

\section{Abstract}

The Multiband Imaging Photometer for SIRTF (MIPS) is to be designed to reach as closely as possible the fundamental sensitivity and angular resolution limits for SIRTF over the 3 to $7 \emptyset \emptyset \mu \mathrm{m}$ spectral region. It will use high performance photoconductive detectors from 3 to $2 \emptyset \emptyset \mathrm{um}$ with integrating JFET amplifiers. From $2 \emptyset \emptyset$ to $7 \emptyset \emptyset \mu \mathrm{m}$, the MIPS will use a bolometer cooled by an adiabatic demagnetization refrigerator. Over much of its operating range, the MIPS will make possible observations at and beyond the conventional Rayleigh diffraction limit of angular resolution.

\section{Introduction}

Our goals with the Multiband Imaging Photometer for SIRTF (MIPS) are to take images with the highest sensitivity and angular resolution that are achievable with SIRTF. To do so, we are developing arrays of detectors with adequate sensitivity to reach the natural background limits for an infrared telescope in earth orbit. These arrays require performance from both the detectors and readouts well beyond that achieved previously. The arrays will be mounted with optical systems that will preserve the image quality of the telescope and set the projected angular pixel diameter to approximately $\boldsymbol{\lambda} / 2.5 \mathrm{D}$, where $\boldsymbol{\lambda}$ is the wavelength of observation and $D$ the telescope aperture. Thus, all spatial frequencies will be sampled to provide fully diffraction limited imaging and to provide imagery at resolutions beyond the conventional diffraction limit with appropriate image processing.

These technical goals will yield an improvement in the sensitivity of infrared imaging by a factor of $1 \emptyset \emptyset \emptyset$ or more over the 4 to $2 \emptyset \emptyset \mu \mathrm{m}$ spectral range, compared with current capabilities. This advance will bring fundamental areas of astronomical research within grasp. For example, we should find a significant number of super massive planets (3 to $6 \emptyset$ times the mass of Jupiter) in orbit around nearby stars, if such objects exist. We will map the global patterns of star formation in nearby galaxies and provide new insights on the processes that trigger and sustain this process. We will be able to study the evolution of galaxies from their birth to the present day, including for the first time the identification of a large number of primeval galaxies. We will follow up on the discoveries of IRAS much as major optical observatories have studied the objects found on the Palomar survey plates.

We begin this paper with a discussion of the technical goals for the MIPS in terms of the limits set by natural backgrounds, confusion, and the characteristics of the SIRTF facility, followed by a description of the instrument itself.

\section{Natural backgrounds}

over the optical and shorter infrared wavelengths, sunlight scattered by zodiacal dust is the dominant background for telescopes in earth orbit. The color of the scattered light is assumed to be similar to that of the sun. The estimates below assume an average scattered zodiacal light of $5 \times 10^{-14} \mathrm{~B}(55 \varnothing \emptyset) 1$, where $\mathrm{B}(\mathrm{T})$ is the blackbody intensity at absolute temperature T. 
From the middle into the far infrared, the dominant natural background is thermal emission by zodiacal dust. We have used the diffuse background data in the IRAS Catalog Explanatory Supplement, Table VI.B.l, to generate a model for the emission near the ecliptic pole of $6.02 \times 10^{-8} \mathrm{~B}(265)$ with a temperature uncertainty of 25 to $30 \mathrm{~K}$. The extrapolated background near $6 \mu \mathrm{m}$ has a significant uncertainty as a result of this temperature uncertainty.

At the longer wavelengths, a contribution to the high galactic latitude background seen by detectors on SIRTF will be made by interstellar dust. This flux has been calculated assuming the Werner-Salpeter interstellar radiation field and a total extinction $A_{\mathrm{v}}=\emptyset . \emptyset 3$ to the galactic pole. The total power absorbed by grains along the line of sight perpendicular to the disk of the galaxy is $6 \mathrm{x} 1 \emptyset-12 \mathrm{~W} / \mathrm{cm}^{2} \mathrm{sr}$. The spectral distribution of this emission depends on the grain temperature and wavelength dependent emissivity. From observations of globules ${ }^{2}$, we would guess that an effective temperature of $15 \mathrm{~K}$ is reasonable, but the IRAS backgrounds indicate a substantially stronger contribution at løøum than this temperature would produce. We have therefore taken the radiation field to be approximately $6 \times 10^{-5} \mathrm{~B}(2 \varnothing)$.

Beyond $2 \emptyset \emptyset$ microns, the telescope contribution to the background is large; we have assumed it to be equivalent to a $5 \mathrm{kgreybody}$ with an emissivity of $2 \emptyset \%$.

Some typical results are given in Table l. General experience indicates that high quality photometry requires a passband of no more than $20 \%$ to $30 \%$ (note the $B, V, R, J, H$, and $\mathrm{K}$ bands); broader bandpasses can involve large and uncertain corrections for spectral characteristics. We therefore quote most of the performance goals for bands of this width.

Table 1. Backgrounds and Required Detector Performance for MIPS

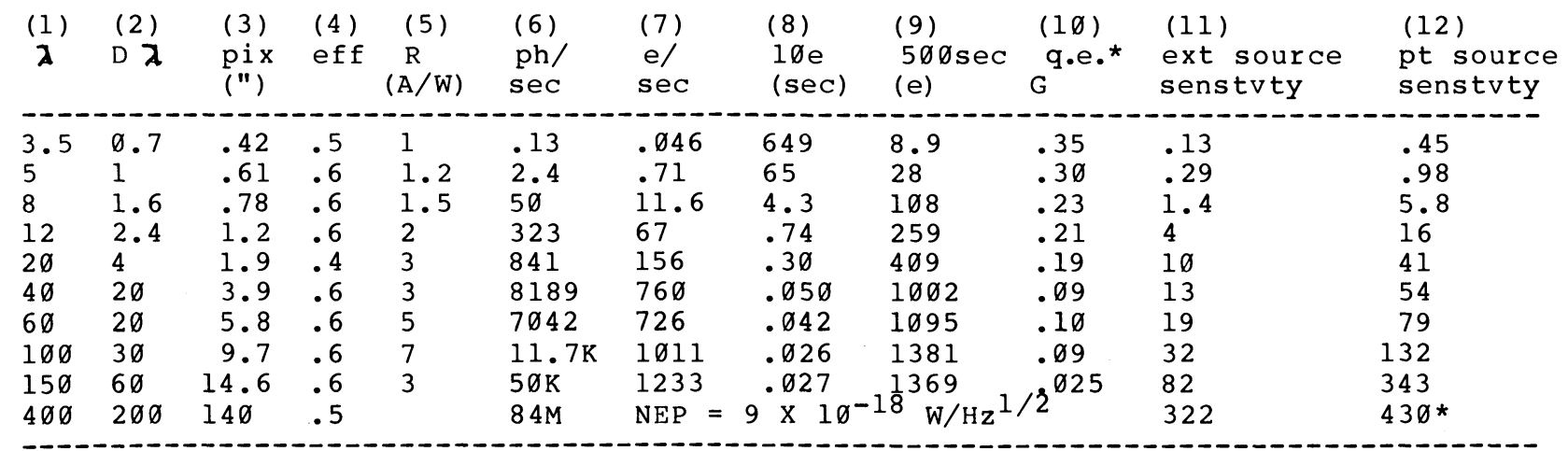

column (1): center wavelength ( $\mu \mathrm{m})$

$(2)$ : passband $(\mu \mathrm{m})$

(3): pixel size in arcsec; $\boldsymbol{\lambda} / 2 \mathrm{D}$ for 3.5 and $5 \mu \mathrm{m} ; \boldsymbol{\lambda} / 2.5 \mathrm{D}$ for 8 through $150 \mu \mathrm{m} ; \boldsymbol{\lambda} / .7 \mathrm{D}$ for $4 \emptyset \emptyset \mu \mathrm{m}$

(4): instrument efficiency, not including telescope or detector

(5): detector responsivity, $\mathrm{A} / \mathrm{W}$

(6): photon rate incident on detector from sum of background sources

(7): photo-current from (6)

(8): seconds of integration required for background noise to reach $1 \emptyset$ e (rms)

(9): read noise required so background noise equals read noise after $5 \emptyset \emptyset$ sec of integration

(10): quantum efficiency times photoconductive gain

(11): one standard deviation signal after $5 \emptyset \emptyset$ seconds integration with $1 \emptyset$ e read noise for an extended (uniform surface brightness) source, expressed in $\mu \mathrm{J} y$.

(12): same as (11), except for a point source centered on a pixel

* Background limited performance listed; MIPS goal is 3 times worse. It is believed that confusion will limit MIPS performance, not background flux.

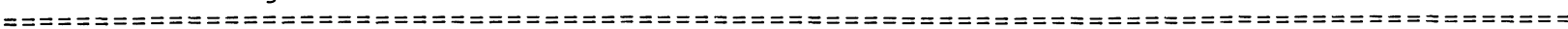

\section{Confusion}

Under some circumstances, the sensitivity of SIRTE may be limited by confusion rather than by detector or background noise. At high galactic latitudes, the dominant types of confusing source are faint red stars in the halo of our galaxy and distant external galaxies. 
We have taken a representative estimate of the density of faint stars within $5 \emptyset^{\circ}$ of the galactic pole and at a longitude $90^{\circ}$ from the galactic center from the work of Bahcall and Soneira ${ }^{3}$. Detailed comparisons indicate good correspondence between their model of the galaxy and the available star counts to very faint limiting magnitudes 4 . The redness of these stars was estimated by computing the absolute magnitude of a star at a given apparent $V$ magnitude and at one scale height $(3 \emptyset \emptyset \mathrm{pc})$ for red dwarfs. The absolute magnitude was then converted into a spectral type and the appropriate V-IR color for that type used to translate the tabulated densities in $V$ magnitudes into equivalent densities in the infrared. Since Bahcall and Soneira tabulated densities down to $V$ magnitudes of 30 , and a $V-K$ cutoff of 6 was imposed, no additional extrapolations in star densities were required.

We have calculated the numbers of galaxies at all wavelengths of interest using the formulation given by Sandage ${ }^{5}$, modified to allow integration over the observed galaxy luminosity function and to include both luminosity and number evolution. Three galaxy types were included. A "normal" galaxy spectrum with no infrared excess was estimated from the published literature for wavelengths shortward of $1 \mu \mathrm{m}$ and from unpublished spectra and photometry 6 of M31, M32, Maffei 1 , and NGC 3115 from 1 through 1øpm. A "starburst" galaxy spectrum was estimated from the observed spectrum of $M 82$, with the excess reduced by a factor of two relative to the direct stellar output. The infrared excess accounts for $8 \emptyset \%$ of the emission of the starburst galaxy. A "spiral excess" galaxy spectrum was taken from published measurements of our galactic plane and of external galaxies with comparatively cool far infrared excesses; the excess radiation was adjusted to provide 50\% of the total emission of this model galaxy. The relative numbers of galaxy type in the local neighborhood were assumed to be $79 \%$ normal, $17 \%$ spiral excess, and $4 \%$ starburst. The optical luminosity functions for all three types were taken from Meurs ${ }^{7}$. The combined number counts of the model galaxies were normalized to the optically measured local density 5 , reduced a factor of two to allow for the local enhancement in the density of galaxies ${ }^{8}$. The percentage of starburst and spiral excess galaxies was adjusted to fit the density of galaxies observed by IRAS at $6 \varnothing \mathrm{um}^{9}$. A variety of calculations were carried out with varying amounts of galactic evolution corresponding to exponential decay in luminosity by factors of 1 to 5 from the epoch $z=5$. Q 0 was varied between $\emptyset$ and $1 . \emptyset$.

We have taken the confusion limit to be at the one standard deviation noise level where there is one chance in three of an accidental source in the measurement beam. At this point, further improvement in the statistical noise will no longer improve the measurement accuracy. Roughly, this definition corresponds to a $2: 1$ detection of a source where there is a $10 \%$ probability of a confusing object, or a 10:1 detection of a source with a $1 \%$ probability of confusion. The uncertainties in our models are substantial, but the calculations indicate clearly that at high galactic latitude SIRTF should not be confusion limited between 8 and $5 \varnothing \mu \mathrm{m}$, that it may be marginally confusion limited shortward of $6 \mu \mathrm{m}$ (where the dominant confusing source is the stellar emission of extremely distant galaxies), and it will be strongly confusion limited longward of $7 \varnothing \mu \mathrm{m}$ (where the dominant source is infrared excess galaxies at reasonably large distance). The source density at $10 \emptyset \mu \mathrm{m}$ is illustrated in Figure 1. With a diffraction limited beam (diameter of $2 / D$ ) at this wavelength, our estimates are that the background limited sensitivity listed in Table 1 will be degraded by confusion by a factor of about five; the effects of confusion become even more serious beyond $1 \emptyset \emptyset \mu \mathrm{m}$.

At the longest wavelengths the dominant source of confusion over most of the sky is likely to be the infrared cirrus. The achievable sensitivity longward of $20 \emptyset \mu \mathrm{m}$ may be limited sufficiently by the cirrus that the background sensitivity limits listed in Table 1 can be relaxed. A study and modeling of the infrared cirrus is planned for the instrument definition study to refine our performance goals for the longest wavelength detectors.

\section{Layout of the MIPS}

The baseline MIPS has three input ports, each fed by a different position of the rotating dichroic beamsplitter in the Multiple Instrument Chamber (MIC). The MIPS is expected to occupy roughly $25 \%$ of the MIC.

The first port will feed a section containing a 16 X 16 array of $\mathrm{Si}: \mathrm{Sb}$ or $\mathrm{Si}: \mathrm{B}$ detectors optimized for super resolution between 13 and $30 \mu \mathrm{m}$. A second array of Si:In will provide for high sensitivity between 3 and $7 \mu \mathrm{m}$. Both arrays will have a common optical system that will include changeable filters and polarizers. The second port will feed a section containing four arrays of germanium photoconductors which together cover the spectral region $3 \emptyset-2 \emptyset \emptyset \mu \mathrm{m}$. The third port serves a section containing an array of bolometers to operate between $2 \varnothing \emptyset$ and $7 \varnothing \emptyset \mu \mathrm{m}$. The basic parameters of the instrument sections are described in Table 2. 
Table 2. Baseline Instrument Parameters for MIPS

\begin{tabular}{|c|c|c|c|c|}
\hline Band $(\mu \mathrm{m})$ & Filters & Polarizers & $\begin{array}{l}\text { Magnification } \\
\text { Change }\end{array}$ & $\begin{array}{l}\text { Fov (pixels or } \\
\text { arcmin) }\end{array}$ \\
\hline $\begin{array}{l}3-7 \\
13-3 \emptyset \\
3 \emptyset-55 \\
5 \emptyset-8 \emptyset \\
8 \emptyset-12 \emptyset \\
12 \theta-2 \theta \emptyset \\
2 \varnothing \emptyset-7 \emptyset \emptyset\end{array}$ & $\begin{array}{l}\text { changeable } \\
\text { changeable } \\
\text { fixed } \\
\text { fixed } \\
\text { fixed } \\
\text { fixed } \\
\text { changeable }\end{array}$ & $\begin{array}{l}\mathrm{Y} \\
\mathrm{Y} \\
\mathrm{Y} \\
\mathrm{N} \\
\mathrm{Y} \\
\mathrm{N} \\
\mathrm{Y}\end{array}$ & $\begin{array}{l}\mathrm{Y} \\
\mathrm{N} \\
\mathrm{N} \\
\mathrm{N} \\
\mathrm{N} \\
\mathrm{N} \\
\mathrm{N}\end{array}$ & $\begin{array}{l}16 \times 16 \\
16 \times 16 \\
16 \times 16 \\
\text { approx } 4{ }^{\prime} \\
\text { approx } 4{ }^{\prime} \\
\text { TBD } \\
\text { TBD }\end{array}$ \\
\hline
\end{tabular}

\section{Detectors and Readouts}

To reach the performance goals outlined in Table 1 , we require the detectors and readouts to operate at a very high level of performance. In particular, the detector dark current per pixel should be less than the photocurrent generated by the backgrounds (column 7). The time to reach the background goes as the square of the read noise, so column 8 demonstrates that very small read noises are required to reach the background in short integrations at wavelengths short of $20 \mu \mathrm{m}$.

IRAS and the Spacelab II Infrared Telescope have led to the development of extrinsic Si and Ge detectors suitable for low background astronomical applications between 8 and $30 \mu m$, where they are based on silicon doped with gallium, arsenic, or antimony, and between $5 \emptyset$ and $126 \mu \mathrm{m}$, where they are based on gallium doped germanium, The latter material is of particular interest for the MIPS; laboratory tests of $1 \mathrm{~mm}^{3}$ detectors demonstrate a peak responsivity of $5 \mathrm{~A} / \mathrm{W}$, dark current of 2500 electrons/sec, and quantum efficiency of $20 \%$. If $0.5 \times 0.5 \times 1 \mathrm{~mm}$ detectors are used for the MIPS, these measurements indicate that the performance goals in Table 1 will be met by this detector material (assuming the dark current scales with detector volume). We are investigating detector geometries that will improve the quantum efficiency, yielding higher sensitivity than predicted by Table l. Tests of the IRAS Si:Sb material show it to have a dark current of about $10 \emptyset$ electrons/second in a $1 \mathrm{~mm}^{3}$ element, also adequate for the requirements in Table 1.

At the time of the design of the IRAS and spacelab II focal planes, no good photoconductor was available for the $3 \emptyset-5$ gum region. Good performance at these wavelengths has recently been demonstrated for Ge:Be 10 compared with Ge:Ga used at the same wavelengths, the Ge:Be material is more sensitive by a factor of about 3 . Further tests of this material show promise in meeting the performance goals in Table 1 , although the best currently available samples have quantum efficiencies of about $1 \emptyset q$ and responsivity of only about $1 \mathrm{~A} / \mathrm{W}$.

Shortward of $7 \mu \mathrm{m}$, we have demonstrated a quantum efficiency of 408 and dark current of about 100 electrons/second with $\mathrm{Si}$ In in $1 \mathrm{~mm}^{2}$ detector elements. Even with the largest feasible reductions in element size, this material falls short of the SIRTF performance requirements unless the dark current path contributes far less noise than the square root of the number of electrons. Continued development is under way on this material.

The cutoff of photoconductive response due to acceptors in Ge can be shifted to longer wavelengths by the application of a uniaxial stress. Operation of Ge:Ga at 2øøj has been achieved by the application of $100 \mathrm{~kg} / \mathrm{mm}^{2}$ stress 11 . Efforts are under way to optimize these detectors for their application in MIPS.

For wavelengths beyond $250 \mu \mathrm{m}$, bolometers are the only practical detectors known. The best low background NEPs reported at ${ }^{3} \mathrm{He}$ temperatures $(0.3 \mathrm{~K})$ are in the mid to $10 \mathrm{w} 1 \emptyset^{-16} \mathrm{~W}$ $\mathrm{Hz}^{-1 / 2}$ range and therefore fall short of the performance required for SIRTF. Extrapolation of bolometer performance with temperature suggests that an operating temperature $\mathrm{T}=0.1 \mathrm{~K}$ will be required for adequate performance. The MIPS therefore includes an adiabatic demagnetization refrigerator to cool the bolometers to this temperature range. Based on our experience with bolometers at $0.3 \mathrm{~K}$ we anticipate that an NEP of about $3 \times 10^{-17}$ will be achieved at $0.1 \mathrm{~K}$. This performance would satisfy the criterion for the long wavelength channels of the MIPS. Adiabatic demagnetization is a classic method for achieving low temperatures, but significant engineering effort in the areas of low current magnets, magnetic shielding, and thermal design is required to adapt it for use in spacecraft.

The response of photoconductors and bolometers and their readouts can be affected by 1 ) the signal power; 2) the background power; 3 ) the history of irradiation by charged particles; and 4) aging of contacts and electrical components. These problems will be addressed explicitly in the design and construction of the MIPS. Charged particle 
irradiation produces undesirable changes in detector response and noise; a boost of the detector bias to induce breakdown can produce a significant degree of recovery, as in IRAS; complete recovery can be achieved virtually instantaneously if the detectors are thermalized by heating them ${ }^{2}$. Both IRAS and SLII IRT use small stimulators to calibrate the relative response of the detectors. These devices proved extremely stable throughout the IRAS mission. Each detector array for the MIPS would have two (for redundancy) of these stimulators built into its mounting hardware in a geometry that would remain undisturbed from initial testing through flight to provide an accurate calibration of the response of the detectors through the life of the instrument.

Readouts for the MIPS detector arrays will be based on the integrating JFET amplifiers under development at steward Observatory and Infrared Laboratories, Inc. 13, 14. The excellent low frequency performance of the JEET permits the signal to be determined by sampling at the beginning and end of the integration; kTC noise is therefore avoided, since the RC time constant for changes in charge is very long while integrating. In fact, since readout of the accumulated charge does not involve alteration or destruction of the charge, this circuit permits unlimited or continuous sampling of the accumulated charge, with resets only as required to prevent saturation. The present version of this circuit can measure noise currents of 3 electrons/Hz $1 / 2$ in integrations of a second or less. The circuit is capable of integrating for twenty seconds with only a slight increase in readout noise. With minimum capacitance detectors, saturation occurs at about $10^{6}$ electrons. These characteristics are easily adequate for the requirements 1 isted in Table 1 for wavelengths longward of $8 \mu m$. Additional work continues on this circuit to reduce its noise, particularly in long integrations required to meet the SIRTF goals at the shortest wavelengths.

\section{Data Analysis}

Because of the modest aperture and very high sensitivity of SIRTF, there is the possibility of using image processing techniques to trade signal to noise for enhanced angular resolution. When the resolution has been boosted beyond the conventional Rayleigh criterion, $1.2 \mathrm{2} / \mathrm{D}$, this procedure is termed super resolution. Super resolution is possible with SIRTF because of a variety of factors: 1.) operation in space removes the effects of atmospheric turbulence; 2.) since SIRTF is to be diffraction limited at wavelengths of 2.5 $4 \mu \mathrm{m}$, the point spread function at the longer wavelengths can be expected to be extremely stable; and 3.) the expected pointing wander is much smaller than the image diameter at the longer wavelengths. The major limitation in applying resolution boosting techniques with SIRTF appears to be the pointing stability of $\emptyset .15$ arcsec rms. Since the relevant parameter is the wander in units of the beam diameter, $2 / D$, this constraint results in a shortest wavelength at which the technique can be applied. This wavelength and other aspects of resolution boosting are functions of the particular algorithm employed, the integration time over which pointing stabilities are allowed to accumulate, and the observational circumstances and scientific goals.

An understanding of the possibilities for super resolution requires the simulation of the expected data and application of test deconvolution algorithms. Although a large number of possible parameters and algorithms need to be explored, for the present we have confined ourselves to two test situations: 1.) two point sources and 2.) a field with a sufficientiy dense distribution of point sources that it is heavily confusion limited. The first test case could correspond to searching for a brown dwarf in orbit around a nearby star and the second case to imaging a deep field at løøum. The deconvolution of these fields has used a simple algorithm based on the CLEAN procedure familiar to radio astronomers. In our case, the algorithm identifies the point of maximum brightness in the simulated data and subtracts a point spread function of amplitude comparable with the rms noise from this position. The point of maximum brightness in the remaining distribution is identified and the procedure repeated until the sum of the squares of the fluxes in the remaining distribution begins to increase. This procedure provides super resolution because it identifies a distribution of point sources that can account for the flux distribution observed.

The signal to noise in super resolution depends strongly on introducing as many constraints as can reasonably be deduced, for example that there are no negative sources, and that the underlying distribution should be the simplest consistent with the observations ${ }^{15}$. In the two scientific objectives mentioned above, we expect the astronomical sources to be point-like -- this is obvious in the case of the brown dwarf search, and we expect the predominant confusing sources at løøum to be distant galaxies with diameters of a few arcsec compared with $\lambda / D$ of 24 arcsec. The CLEAN method of resolution boosting makes this assumption implicitly and should be a reasonably good procedure to use in these situations.

Our tests consisted of generating an appropriate two dimensional distribution of point sources in a $2 \emptyset \times 2 \emptyset$ array; in the confusion limited case, $4 \emptyset \emptyset$ sources were drawn froma $1 / f$ distribution where $f$ is the source brightness. This distribution was convolved with an assumed beam profile and random noise drawn from a gaussian distribution was added. To allow 
for sources that might fall just outside the area observed, the CLEAN algorithm was then applied to the central $16 \times 16$ area of the array, and the results were compared with the original distribution both by inspection of contour maps and by a $\mathbf{x}$-square test.

Two general conclusions resulted from our study. First, we found that the restoration near the edge of an assumed image tended to be unreliable. The reduced $X$-square computed for the entire restored image was typically 2 to 3 times larger than that for the central $12 x$ 12 section of the image. One consequence is that studies of super resolution based on one dimensional source distributions will yield optimistic results, since the "edge" is confined to a few pixels. Second, lower degrees of image boosting were possible with the correct diffraction limited beam profile than with a gaussian profile as is frequently used in simulations. This difference is subtle at restorations up to about $\boldsymbol{\lambda} / 2 \mathrm{D}$ resolution, but becomes increasingly important at higher degrees of super resolution. The effect is easy to understand, since the diffraction limited beam profile cuts off the spatial frequencies completely at an upper limit corresponding to the telescope aperture, whereas a gaussian contains frequencies beyond this limit.

We also reached a number of specific conclusions regarding super resolution with SIRTF. First, we found that an uncompensated guiding drift less than $\emptyset .1 \boldsymbol{\lambda} / \mathrm{D}$ had little effect on the reliability of the image restorations; as the drift increased beyond this limit, the effects on the restoration grew rapidly. Interpreting this limit as a two-standard-deviation limit on the acceptable drift, the guiding stability of $\varnothing .15$ arcsec rms implies that super resolution should be effective at wavelengths of $13 \mu \mathrm{m}$ and longer. Second, resolution boosting to $\lambda / 2 \mathrm{D}$ should be effective in heavily confusion limited fields, even when they are observed at modest signal to noise. Figure 2 shows an example, in which the assumed rms noise for each pixel is $1 \varnothing \%$ of the total brightness of the brightest sources. Consequently, super resolution will increase the useful sensitivity of SIRTE in imaging confusion limited fields, such as deep exposures at løøum. Third, resolution boosting to beyond $\lambda / 3 D$ was unceliable at any signal to noise. We suspect that this result is a consequence of the edge effects mentioned earlier, since these appear to increase rapidly as the resolution is increased. If this hypothesis is correct, higher degrees of resolution boost will be possible where larger areas on the sky are observed or where the source structure is more centrally concentrated. Fourth, CLEAN was effective at distinguishing two point sources with a brightness contrast of $10 \emptyset: 1$, so long as the weaker source was detected at a signal to noise of at least $5: 1$ and was at a distance of at least $0.6 \lambda / D$ from the primary. Better algorithms may improve on these results, but our study has already established the general usefulness of super resolution in at least two important types of observation.

\section{Conclusion}

The MIPS is being designed to provide sensitivity and angular resolution limited only by natural backgrounds and the telescope design over the entire operating spectral range of SIRTF, 3 to $7 \emptyset \emptyset$ um. The requisite detector and readout performance for most of this spectral range has already been achieved; development is continuing for the 3 to 7 and $2 \emptyset \emptyset$ to $7 \emptyset \emptyset \mu m$

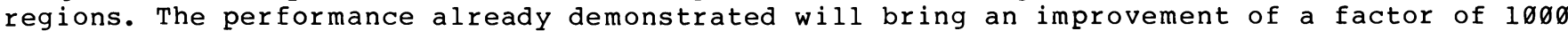
over current imaging capabilities between 8 and $2 \emptyset \emptyset \mu \mathrm{m}$.

Acknowledgements

We thank the Martin Marietta Corporation for assistance in preparing the initial concept for the MIPS. The calculations for super resolution were carried out by Mr. B. Sharma. This work was supported by NASA.

\section{References}

1. Allen, C. W. 1963, Astrophysical Quantities, (London, Athlone Press).

2. Keene, J., Davidson, J. A., Harper, D. A., Hildebrand, R. H., Jaffe, D. T., Loewenstein, R. F., Low, F. J., and Pernic, R. 1983, Ap. J. (Lett.), 274, L43.

3. Bahcall, J. N., and Soneira, R. M. 1980, Ap. J. Suppl., 44, 73.

4. Bahcall, J. N., and Soneira, R. M. 1983, IAU Colloq. No. 76, 209.

5. Sandage, A. 1961, Ap. J., 134, 916.

6. Lebofsky, M. J., and Rieke, G. H., unpublished.

7. Meurs, E. J. A. 1982, unpublished Ph.D. thesis.

8. Tyson, J. A., and Jarvis, J. F. 1979, ApJ (Lett), 230, L153.

9. Boggess, N. 1983, "SIRTF Announcement of Opportunity Pre-proposal Conference".

10. Haegel, N. M., Haller, E. E., Hansen, W. L., Hueschen, M. R., Luke, P. N., Richards, P. L., Townes, C. H., and Watson, D. M. 1983, Int. J. IR and MM Waves, in press.

11. Haller, E. E., Richards, P. L., and Hueschen, M. 1979, Appl. Phys. Lett., $34,495$.

12. Low, F. J. 1984, Applied Optics, 23, 1308 .

13. Low, F. J., and Alwardi, M. 1985, 2nd Infrared Technology Workshop, ed. McCreight, in press.

14. Young, E. T. 1983, Adv. Space Res., 2, 59.

15. Frieden, B. Roy 1972, J. O. S. A., 62, 511 . 
Figure 1. Source density at $1 \varnothing \emptyset \mu \mathrm{m}$ vs. flux. The integral number of sources per degree is plotted vs. the flux limit in Jy. The solid line is for galaxy evolution by a factor of 5 from $z=5$ and the dashed line is for no evolution. In both cases, $q_{0}=\emptyset .3$.

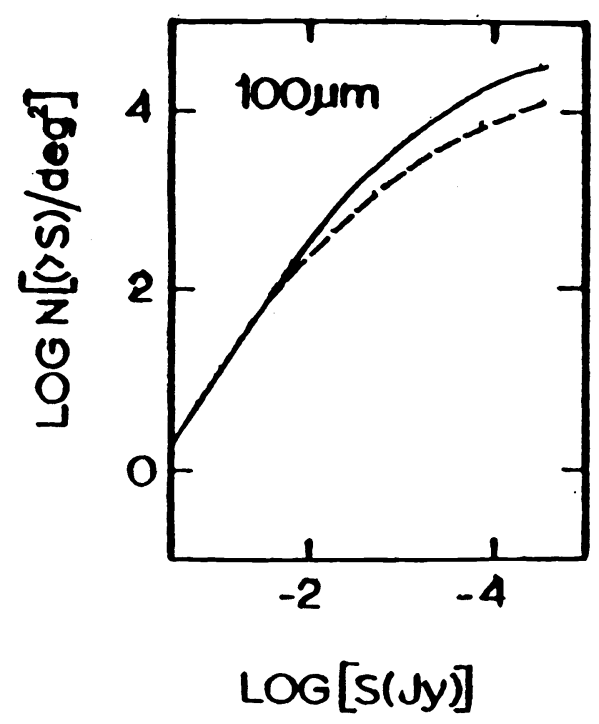

Figure 2. Super resolution in a confused field. The upper left panel is the original source distribution. In the upper right one, it has been convolved with a beam that degrades its resolution by a factor of two, and a noise of $10 \%$ rms the strength of the brightest source has been added to each pixel. The lower panel shows the results of deconvolution of the upper right panel using CLEAN.
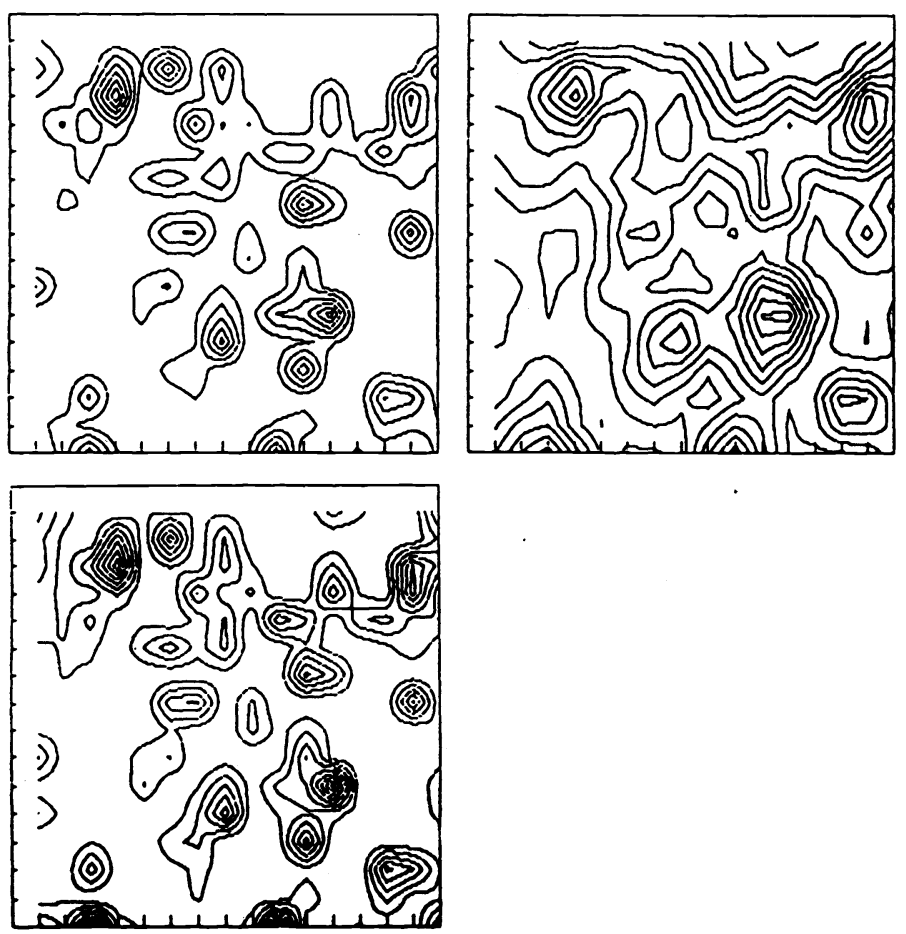